\title{
Milliliter per 100 Grams per Minute
}

National Cancer Institute

\section{Source}

National Cancer Institute. Milliliter per 100 Grams per Minute. NCI Thesaurus. Code C154855.

A unit of flow rate expressed as the number of milliliters per 100g of material per minute. 